\title{
Multidrug-Resistant (MDR) Acinetobacter: a Major Nosocomial Pathogen Challenging Physicians
}

\author{
Shahina Tabassum \\ Department of Virology, Bangabandhu Sheikh Mujib Medical University (BSMMU), Shahbag, Dhaka-1000
}

\section{Introduction}

Acinetobacter species are becoming a major cause of nosocomial infections and can present major challenges for physicians. Of growing concern is the increase in multidrug resistance exhibited by clinically relevant species. $A$. baumannii is emerging as a cause of numerous global outbreaks, displaying ever-increasing rates of resistance. ${ }^{1}$ Today, the increasing recovery of multi-drug resistant (MDR) A. baumannii in the nosocomial setting is a frightening reality. ${ }^{2}$

\section{The Organism}

Acinetobacter are pleomorphic, encapsulated, aerobic, nonfermentative gram-negative bacilli (similar in appearance to Haemophilus influenzae on Gram stain). Acinetobacter species are ubiquitous, living in soil and water. The organism can survive for extended periods in the environment and tolerates both wet and dry conditions. ${ }^{3}$ Acinetobacter utilizes a wide variety of carbon and other energy sources and grows well on routine laboratory media. A tendency to retain crystal violet in the cell wall of the organism can lead to incorrect identification as gram-positive cocci. ${ }^{4}$ There are over 20 species of Acinetobacter, although the species A. baumannii accounts for $>80 \%$ of isolates causing human disease. ${ }^{4}$ The organism can survive for months on clothing and bedclothes, bed rails, ventilators and other surfaces in the environment, including sinks and doorknobs, making nosocomial transmission extremely difficult to control.

\section{Epidemiology}

Acinetobacter is an organism of low virulence, but it is capable of causing infections. A. baumannii is primarily a healthcare-associated pathogen commonly isolated from the hospital environment and hospitalized patients. It is increasingly reported as the cause of outbreaks and nosocomial infections such as blood-stream infections, ventilator-associated pneumonia (VAP), urinary tract infections (UTI) and wound infections. 5,6 Most Acinetobacter isolates recovered from hospitalized patients, particularly those recovered from specimens of respiratory secretions and urine, represent colonization rather than infection.

Acinetobacter isolates demonstrate increasing resistance to commonly prescribed antimicrobials. ${ }^{7}$ Multidrug-resistant $A$. baumannii has been reported worldwide and is now recognized as one of the most difficult healthcare-associated infections to control and to treat. ${ }^{5}$ Multidrug-resistant Acinetobacter is not a new or emerging phenomenon, but $A$. baumannii has always been an organism inherently resistant to multiple antibiotics. A citywide clonal outbreak in New York City reported that $53 \%$ of $A$. baumannii were resistant to carbapenems while $12 \%$ of them were resistant to all standard antimicrobial agents. ${ }^{8}$

Multidrug-resistant Acinetobacter rarely causes serious infection in otherwise healthy people, and therefore, poses minimal threat to healthcare workers or patients' family members. On the other hand, MDR A. baumannii infections tend to occur in immunosuppressed patients, in patients with serious underlying diseases, and in those subjected to invasive procedures and treated with broad spectrum antibiotics. 9 Thus, infections due to $A$. baumannii are frequently found in intensive care units, where they are implicated as the cause of VAP, UTIs and bacteremia. Less frequently, A. baumannii also causes complicated skin and soft tissue, abdominal, and central nervous system infections. ${ }^{10}$ Of recent importance, A. baumannii has become a major pathogen found in combat-associated wounds. ${ }^{11}$ 
Outbreaks are frequently located in intensive-care units and burn units involving patients on mechanical ventilation. ${ }^{6}$ Sources of transmission identified in the outbreak setting include predominately respiratory equipments such as resuscitator bags, valves, ventilator circuits, spirometers, peak flow meters, suction catheters, etc. Other sources include humidifiers, warming baths, multidose vials, distilled water, pillows, mattresses, bedpans, showers and water faucet aerators. No source was identified in approximately $50 \%$ of reported outbreaks. ${ }^{6}$ The factors contributing to colonization, virulence, and invasion are being defined. ${ }^{12}$ It is often difficult to distinguish between infection and colonization with $A$. baumannii. ${ }^{13}$ Risk factors for colonization or infection with multidrug-resistant Acinetobacter include length of hospital stay, surgery, wounds, treatment with broad-spectrum antibiotics, parenteral nutrition, indwelling catheters, mechanical ventilation, and admission to an intensive care unit. ${ }^{4}$

\section{Diagnosis}

Infection or colonization with Acinetobacter is usually diagnosed by clinical culture of properly transported, plated, and incubated blood, sputum, urine, wound, sterile body fluid, etc. Recovery of the organism from a non-sterile body site (e.g., endotracheal secretions, urine in patient with Foley's catheter) does not indicate or imply an infectious pathogenic role. In outbreaks, Acinetobacter may be easily cultured from monitoring devices or biological fluids from multiple patients as part of an epidemiological investigation.

Antimicrobial susceptibility can be determined by various means, with the agar-dilution method being the goldstandard. Definitions for "multidrug-resistance" vary widely in the published literature. Multidrug-resistant Acinetobacter is defined as an isolate that is susceptible to no more than one class of antimicrobial agents, excluding colistin. (e.g., most isolates that meet the definition are completely resistant to commonly prescribed antibiotics or are susceptible to only the aminoglycoside class of agents).

\section{Treatment}

Regretably, there are few antibiotics for the treatment of infections caused by MDR A. baumannii as this organism is intrinsically multidrug resistant. Relatively few antibiotics are effective for the treatment of severe nosocomial Acinetobacter infections, particularly in patients confined to ICUs. But it should be noted that colonization not to be treated, only infections should be treated. Carbapenems (imipenem and meropenem) are the mainstay of treatment for antimicrobial-resistant gram-negative infections, though carbapenem-resistant Acinetobacter is increasingly reported. ${ }^{8}$ Resistance to the carbapenem class of antibiotics makes multidrug-resistant Acinetobacter infections difficult, if not impossible, to treat. Colistin and polymyxin B have been used to treat highly resistant Acinetobacter infections. ${ }^{14,15}$ The choice of appropriate therapy is further complicated by the toxicity of colistin which is mainly renal. ${ }^{15}$ Acinetobacter isolates resistant to colistin and polymyxin $\mathrm{B}$ have also been reported. ${ }^{16,17}$ In general, first-, second-, and third-generation cephalosporins, macrolides, and penicillins have little or no anti-Acinetobacter activity, and their use may predispose to Acinetobacter colonization. Among the antibiotics that are considered as agents against MDR A. baumannii, tigecycline has received significant attention. Tigecycline has shown excellent in vitro activity against multiple clinical isolates of A. baumannii. ${ }^{18,19}$ Doripenem, a novel carbapenem, also promises to be active against susceptible $A$. baumannii. ${ }^{20,21}$ Studies have demonstated in vitro susceptibility of multidrugresistant Acinetobacter to various synergistic combinations of antimicrobials including carbapenems, colistin, rifampin, and ampicillin-sulbactam. ${ }^{15,22,23}$ The clinical utility of these combinations against panresistant Acinetobacter remains to be determined.

\section{Prevention}

The costs associated with control of an outbreak can be staggering, and some institutions have been forced to close entire units in order to interrupt transmission of Acinetobacter.5,24 Therefore, there is great incentive to prevent transmission in the healthcare setting and keep the organism from becoming endemic in an institution. For this, infection control is crucial, particularly given the ability of $A$. baumannii to cause outbreaks. As with prevention of any healthcare-associated organism, careful hand hygiene should be performed at all appropriate times - either hand washing at the sink or using an alcohol based hand sanitizer. Standard precautions should be followed at all times. The utility of active surveillance cultures to detect patients who are colonized with multidrug-resistant Acinetobacter remains to 
be determined. Measures reported to be successful in control of outbreaks include isolation precautions for infected or colonized patients, reinforcement hand hygiene, cohorting of patients, cohorting of staff, environmental disinfection, antimicrobial control, and unit closure. ${ }^{6}$ The success of these and other approaches for the containment of MDR $A$. baumannii depends on the commitment of clinical practitioners, scientists, hospital and public health administrators, and on the support of an informed and concerned public.

\section{References}

1. Bergogne-Berezin E, Towner KJ. Acinetobacter spp. as nosocomial pathogens: microbiological, clinical, and epidemiological features. Clin Microbiol Rev 1996; 9: 148-165.

2. Livermore DM. The threat from the pink corner. Ann Med 2003; 35: $226-234$

3. Jawad A, Heritage J, Snelling AM, Gascoyne-Binzi DM, Hawkey PM. Influence of relative humidity and suspending menstrua on survival of Acinetobacter spp. on dry surfaces. J Clin Microbiol 1996; 34 (12): 2881-2887.

4. Allen D, Hartman B. Acinetobacter Species. In: Mandell GL, Bennett JE, Dolin R, editors. Mandell, Douglas, and Bennett's Principles and Practice of Infectious Diseases. Philadelphia: Churchill Livingstone; 2000: pp. 2339-2344.

5. Urban C, Segal-Maurer S, Rahal JJ. Considerations in control and treatment of nosocomial infections due to multidrugresistant Acinetobacter baumannii. Clin Infect Dis 2003; 36 (10): 1268-1274.

6. Villegas MV, Hartstein AI. Acinetobacter outbreaks, 1977-2000. Infect Control Hosp Epidemiol 2003; 24 (4): 284-295.

7. Wisplinghoff H, Edmond MB, Pfaller MA, Jones RN, Wenzel RP, Seifert H. Nosocomial bloodstream infections caused by Acinetobacter species in United States hospitals: clinical features, molecular epidemiology, and antimicrobial susceptibility. Clin Infect Dis 2000; 31 (3): 690-697.

8. Landman D, Quale JM, Mayorga D, et al. Citywide clonal outbreak of multiresistant Acinetobacter baumannii and Pseudomonas aeruginosa in Brooklyn, NY: the preantibiotic era has returned. Arch Intern Med 2002; 162 (13): 1515-1520.

9. Garcia-Garmendia JL, Ortiz-Leyba C, Garnacho-Montero J, et al. Risk factors for Acinetobacter baumannii nosocomial bacteremia in critically ill patients: a cohort study. Clin Infect
Dis 2001; 33: 939-346.

10. Fournier PE, Richet H. The epidemiology and control of Acinetobacter baumannii in health care facilities. Clin Infect Dis 2006; 42: 692-699.

11. Aronson NE, Sanders JW, Moran KA. In harm's way: infections in deployed American military forces. Clin Infect Dis 2006; 43: 1045-1051.

12. Smith MG, Gianoulis TA, Pukatzki S, et al. New insights into Acinetobacter baumannii pathogenesis revealed by highdensity pyrosequencing and transposon mutagenesis. Genes Dev 2007; 21: 601-614

13. Joly-Guillou ML. Clinical impact and pathogenicity of Acinetobacter. Clin Microbiol Infect 2005; 11: 868-873.

14. Levin AS, Barone AA, Penco J, et al. Intravenous colistin as therapy for nosocomial infections caused by multidrug-resistant Pseudomonas aeruginosa and Acinetobacter baumannii. Clin Infect Dis 1999; 28 (5): 1008-1011.

15. Giamarellos-Bourboulis EJ, Xirouchaki E, Giamarellou H. Interactions of colistin and rifampin on multidrug-resistant Acinetobacter baumannii. Diagn Microbiol Infect Dis 2001; 40 (3): $117-120$

16. Manikal VM, Landman D, Saurina G, Oydna E, Lal H, Quale J. Endemic carbapenem-resistant Acinetobacter species in Brooklyn, New York: citywide prevalence, inter-institutional spread, and relation to antibiotic usage. Clin Infect Dis 2000; 31 (1): 101-106.

17. Rhomberg PR, Jones RN, Sader HS. Results from the Meropenem Yearly Susceptibility Test Information Collection (MYSTIC) Programme: report of the 2001 data from 15 United States medical centres. Int J Antimicrob Agents 2004; 23(1): 52-59.

18. Jones RN, Ferraro MJ, Reller LB, Schreckenberger PC, Swenson JM, Sader HS. Multicenter studies of tigecycline disk diffusion susceptibility results for Acinetobacter spp. J Clin Microbiol 2007; 45: 227-230.

19. Waites KB, Duffy LB, Dowzicky MJ. Antimicrobial susceptibility among pathogens collected from hospitalized patients in the United States and in vitro activity of tigecycline, a new glycylcycline antimicrobial. Antimicrob Agents Chemother 2006; 50: 3479-3484.

20. Mushtaq S, Ge Y, Livermore DM. Comparative activities of doripenem versus isolates, mutants, and transconjugants of Enterobacteriaceae and Acinetobacter spp. with characterized 
beta-lactamases. Antimicrob Agents Chemother 2004; 48: 13131319.

21. Jones RN, Sader HS, Fritsche TR. Comparative activity of doripenem and three other carbapenems tested against Gramnegative bacilli with various beta-lactamase resistance mechanisms. Diagn Microbiol Infect Dis 2005; 52: 71- 74.

22. Higgins $\mathrm{PG}$, Wisplinghoff $\mathrm{H}$, Stefanik $\mathrm{D}$, Seifert $\mathrm{H}$. In vitro activities of the beta-lactamase inhibitors clavulanic acid, sulbactam, and tazobactam alone or in combination with betalactams against epidemiologically characterized multidrugresistant Acinetobacter baumannii strains. Antimicrob Agents
Chemother 2004; 48 (5): 1586-1592.

23. Yoon J, Urban C, Terzian C, Mariano N, Rahal JJ. In vitro double and triple synergistic activities of Polymyxin B, imipenem, and rifampin against multidrug-resistant Acinetobacter baumannii. Antimicrob Agents Chemother 2004; 48 (3): 753-757.

24. Simor AE, Lee M, Vearncombe M, et al. An outbreak due to multiresistant Acinetobacter baumannii in a burn unit: risk factors for acquisition and management. Infect Control Hosp Epidemiol 2002; 23 (5): 261-267. 